\title{
Overexpression of calcyphosine is associated with poor prognosis in esophageal squamous cell carcinoma
}

\author{
FENG LI, DENGYAN ZHU, YANG YANG, KAI WU and SONG ZHAO
}

Department of Thoracic Surgery, First Affiliated Hospital of Zhengzhou University, Zhengzhou, Henan 450052, P.R. China

Received May 25, 2016; Accepted July 27, 2017

DOI: $10.3892 / 01.2017 .6973$

\begin{abstract}
Calcyphosine (CAPS), a calcium-binding protein, has been identified as a potential diagnostic and prognostic biomarker in several human carcinomas. However, little is known about CAPS in esophageal squamous cell carcinoma (ESCC). The present study aimed to investigate the expression levels of CAPS in ESCC tissues and evaluate its clinicopathological significance. Reverse transcription-quantitative polymerase chain reaction and immunohistochemical staining were conducted to detect the expression of CAPS in ESCC tissues and adjacent non-cancerous tissues. ESCC samples exhibited higher levels of CAPS mRNA than paired non-cancerous samples $(\mathrm{P}=0.0015)$, and the mRNA level of CAPS was positively associated with histological grade $(\mathrm{P}=0.0013)$ and tumor invasion depth $(\mathrm{P}=0.0206)$. In addition, Kaplan-Meier survival analysis revealed that patients with high CAPS expression experienced significantly shorter 5-year overall survival times than those with low CAPS expression ( $\mathrm{P}=0.0112)$. Multivariate analysis demonstrated that CAPS protein expression was an independent prognostic biomarker for patients with ESCC. In conclusion, the findings of the present study demonstrated that CAPS may represent a novel diagnostic indicator and an independent prognostic biomarker in ESCC.
\end{abstract}

\section{Introduction}

Esophageal cancer is the eighth most common cancer and the sixthleading cause of cancer-associated mortality worldwide (1). Approximately $70 \%$ of global esophageal cancer cases occur in China, with esophageal squamous cell carcinoma (ESCC) accounting for the vast majority of cases (>90\%) (2). Currently, multi-modality treatment improves the quality of life and prolongs the survival time of patients with ESCC. However, the 5-year survival rate remains poor owing

Correspondence to: Professor Song Zhao, Department of Thoracic Surgery, First Affiliated Hospital of Zhengzhou University, 1 Jianshe Road, Zhengzhou, Henan 450052, P.R. China

E-mail: zhaosong@zzu.edu.cn

Key words: calcyphosine, calcium-binding protein, esophageal squamous cell carcinoma, prognosis to the limited clinical opportunities for the early diagnosis and treatment of ESCC. Therefore, there is an urgent requirement to pursue novel diagnostic indicators, prognostic biomarkers, therapeutic targets and therapeutic approaches for ESCC treatment.

Calcyphosine (CAPS), a $\mathrm{Ca}^{2+}$-binding protein, was initially isolated from the canine thyroid cDNA library as a substrate that can be phosphorylated by protein kinase A in a Cyclic adenosine monophosphate (cAMP)-dependent manner $(3,4)$. CAPS was also detected in humans and other mammals such as cows and rabbits, and even in certain invertebrates, such as sponges; however, it was determined to be absent from mice and five other rodents (5-7). To date, three subtypes of CAPS shave been reported: Type-I CAPS, type-II CAPS and CAPS 2. A previous study revealed that type-I CAPS may be specific to mammals, type-II CAPS widely exists in metazoan speciesand CAPS 2 is unique to human beings (8). The synthesis and phosphorylation of type-I CAPS are upregulated by thyrotropin and cyclic AMP analogues that can promote cell proliferation and maintain expression of the differentiated thyrocyte phenotype, and are downregulated by 12-O-tetradecanoylphorbol-13-acetate (TPA) and epidermal growth factor, which repress cell differentiation (9). As a member of the EF hand motif family, CAPS contains four EF-hand domains for calcium binding (10). Although the exact function of CAPS remains unclear, its $\mathrm{Ca}^{2+}$-binding phosphorylatory abilities may implicate it in cross-signaling between calcium-phosphatidylinostitol and cAMP cascades (11).

In recent years, attention has been drawn to the associations between CAPS protein expression and various diseases, including certain types of cancer. For example, CAPS was significantly down regulated in the bronchoalveolar lavage fluid of sulfur mustard-exposed patients when compared with healthy controls (12). Previous studies also showed that CAPS was overexpressed in ovarian cancer (13), ependymoma (14), endometrial cancer (15), lung cancer (16) and colorectal cancer (17). Another previous study revealed that CAPS promoted cancer progression and may be a prognostic indicator in colorectal cancer patients (17). However, the expression and role of CAPS in ESCC require further investigation. The present study investigated CAPS expression in ESCC tumor tissues, and examined the association between CAPS expression, clinicopathological features and survival outcomes for patients with ESCC. To the best of our knowledge, this is the first study of the clinical relevance of CAPS in ESCC to date. 


\section{Materials and methods}

Ethics statement. This study was approved by the Ethics Committee of the First Affiliated Hospital of Zhengzhou University (Zhangzhou, China) and written informed consent was obtained from each patient involved in the present study.

Tumor samples. A total of 104 fresh samples of tumor tissues were immediately harvested from patients ( 40 women, 64 men; mean age, 62.36 years and range $42-80$ years) with ESCC who underwent surgical resections between November 2013 and January 2015 in the Department of Thoracic Surgery, the First Affiliated Hospital of Zhengzhou University. None of the patients had received preoperative chemotherapy or radiotherapy. Tumor tissue samples were obtained from operative specimens, washed twice with PBS and maintained in RNA wait (Beijing Solarbio Science \& Technology Co., Ltd., Beijing, China) at $-80^{\circ} \mathrm{C}$ until the time of analysis, following evaluation by a pathologist. Clinical and pathological characteristics were obtained from clinical database and pathology records. A total of 64 formalin-fixed paraffin-embedded ESCC tissues and 4 corresponding adjacent non-cancerous tissues with available follow-up information were obtained from the Department of Pathology, the First Affiliated Hospital of Zhengzhou University between October 2008 and December 2010 , and were used for immunohistochemical analysis. The clinicopathological features were analyzed according to age, gender, tumor invasion depth, histological grade, lymph node metastasis and Tumor-Node-Metastasis (TNM) stage (18).

Reverse transcription-quantitative polymerase chain reaction $(R T-q P C R)$. Total RNA was extracted from ESCC tissue specimens using TRIzol reagent (Invitrogen; Thermo Fisher Scientific, Inc., Waltham, MA, USA) according to the manufacturer's instructions. First-strand cDNA was synthesized from $1 \mu \mathrm{g}$ of total RNA using the Revert Aid First Strand c-DNA Synthesis kit (Fermentas; Thermo Fisher Scientific, Inc., Pittsburg, PA, USA). Briefly, $1 \mu \mathrm{g}$ total RNA samples were incubated at $42^{\circ} \mathrm{C}$ with $2 \mu 15 \mathrm{X}$ gDNA eraser buffer, $1 \mu 1$ gDNA eraser and RNase-free $\mathrm{dH}_{2} \mathrm{O}$ for $2 \mathrm{~min}$, then the enzyme mix was added and the solution was incubated at $37^{\circ} \mathrm{C}$ for $15 \mathrm{~min}$. The CAPS mRNA levels were quantified in duplicate using a Stratagene Mx3005P (Agilent Technologies, Santa Clara, California, USA). The Premix Tap kit (Takara Bio, Inc., Otsu, Japan) was used to perform the qPCR reaction according to the manufacturer's instruction; GAPDH was used as a loading control. PCR thermocycling conditions were as follows: Incubation at $95^{\circ} \mathrm{C}$ for $2 \mathrm{~min}$ followed by 40 cycles of denaturation at $96^{\circ} \mathrm{C}$ for $15 \mathrm{sec}$ and annealing at $60^{\circ} \mathrm{C}$ for $1 \mathrm{~min}$. Eachsample was obtained from three independent experiments and used for analysis of relative mRNA expression normalized by GAPD Husing the $2^{-\Delta \Delta \mathrm{Cq}}$ method (19). The synthetic primers for CAPS and GAPDH were obtained from Sangon Biotech (Shanghai, China) and the primers sequences are shown in Table I.

CAPS staining. For CAPS staining, 4-mm sections of formalin-fixed paraffin embedded tissues were cut and stained. The slides were heated in an oven at $65^{\circ} \mathrm{C}$ for $30 \mathrm{~min}$, deparaffinized in dimethyl benzene for 5 min twice, rehydrated
Table I. Primers used for quantitative polymerase chain reaction.

\begin{tabular}{llc}
\hline Genes & \multicolumn{1}{c}{ Primers } & Product, bp \\
\hline CAPS & \multicolumn{2}{c}{190} \\
Forward & 5'-AGGCACCTTCCACTAGC & \\
& $\begin{array}{l}\text { AACAG-3' } \\
\text { Reverse }\end{array}$ & $\begin{array}{l}\text { 5'-CCATGCTTGGTCTGGGC } \\
\text { TCT-3' }\end{array}$ \\
GAPDH & 5'-AAGGTCATCCCTGAGCT & 271 \\
Forward & 5'-AA-3' & \\
Reverse & 5'-TGACAAAGTGGTCGTTG & \\
& AGG-3' & \\
\hline
\end{tabular}

CAPS, calcyphosine; GAPDH, glyceraldehyde 3-phosphate dehydrogenase.

in graded alcohol $(100,100,95,95,70,70,50$ then $50 \%)$ for 5 min each in turn and washed with TBST. Following antigen retrieval, antigenicity was performed by heating the tissue in citrate buffer; tissues were then blocked with $3 \% \mathrm{H}_{2} \mathrm{O}_{2}$ for $30 \mathrm{~min}$ at room temperature followed by incubation with $10 \%$ goat serum (Santa Cruz Biotechnology, Inc., Dallas, TX, USA) for another $30 \mathrm{~min}$ at room temperature. Samples were then incubated with CAPS primary antibody (cat. no. ab186741, 1:200 dilution; Abcam, Cambridge, UK) in dark box at $4^{\circ} \mathrm{C}$ overnight, followed by treatment with secondary antibody (cat. no. sc2040; IgG-B, 1:200 dilution; Santa Cruz Biotechnology, Inc.) for $15 \mathrm{~min}$ at $37^{\circ} \mathrm{C}$. For the negative control, samples were incubated with PBS instead of specific antibody. Following washing with PBS 4 times, the sections were incubated with biotinylated horseradish peroxidase-labeled streptavidin (Origene Technologies, Inc., Beijing, China) at $4^{\circ} \mathrm{C}$ overnight. The reaction was visualized with 3,3-diaminobenzidine (DAB; Origene Technologies, Inc.) as a peroxidase substrate and the sections were counter-stained with Meyer's hematoxylin at room temperature for $3 \mathrm{~min}$. The slides were visualized using a bright-field light microscope, and at least 5 consecutive non-overlapping fields were viewed (magnification, x200). The detection of nuclear and/or cytoplasmic staining in any percentage of tumor cells was considered positive. Complete absence of staining was considered as negative for CAPS. Immunostaining was scored by two independent pathologists according to the immunoreactive score (IRS). The proportion of positive tumor cells examined was scored as 0 (no positive tumor cells), $1(1-40 \%), 2(41-75 \%)$ or $3(>75 \%)$. Staining intensity was scored as 0 (no staining), 1 (week staining), 2 (intermediate staining) or 3 (strong staining). The stain signal was evaluated by the multiplication values (IRS) of the two scores.

Statistical analysis. Data are expressed as the mean \pm standard error of the mean. Statistical analyses were performed using SPSS 22.0 software (IBM Corp., Armonk, NY, USA) and the GraphPad Prism 5.0 software package (GraphPad Software, Inc., La Jolla, CA, USA). A paired t-test was applied to compare the difference in CAPS expression between tumor tissues and 
A

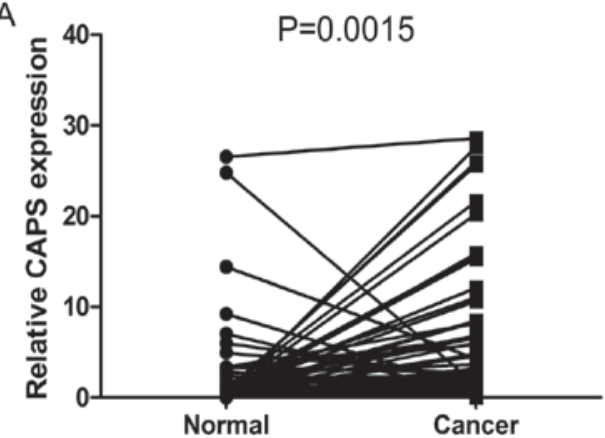

C

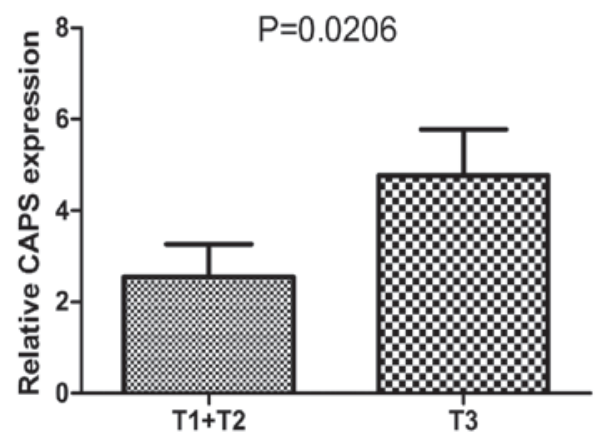

B

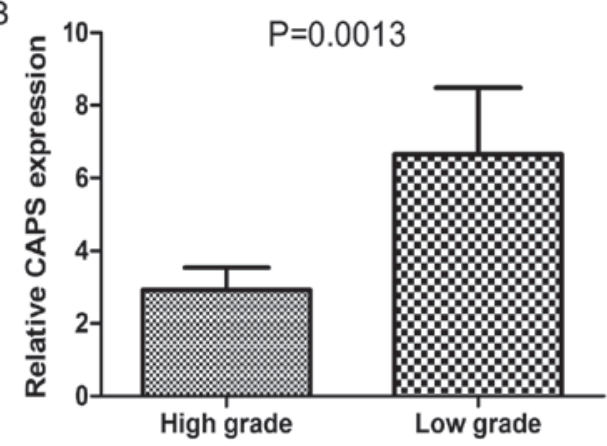

Figure 1. CAPS mRNA expression is upregulated in ESCC tissues. (A) Relative CAPS mRNA levels were measured by reverse transcription-quantitative polymerase chain reaction in 104ESCC tissues and paired adjacent normal tissues. (B) CAPS mRNA levels in ESCC tissues of different histological grades. (C) CAPS mRNA levels in ESCC tissues at different tumor invasion depths. Data are represented as the mean \pm standard error of the mean. CAPS, calcyphosine; ESCC, esophageal squamous cell carcinoma.

adjacent normal tissues. A Mann-Whitney U test was used to compare other groups with one another in terms of CAPS expression. The associations between CAPS expression and clinicopathological parameters of ESCC patients were analyzed via $\chi^{2}$ and Fisher's exact probability tests. The Kaplan-Meier method and log-rank test were used where indicated to plot the overall survival curve and analyze the association of patient survival with CAPS expression. In addition, univariate and multivariate analyses were conducted to evaluate the prognostic value of CAPS expression in patients with ESCC using the Cox proportional hazards regression model. $\mathrm{P}<0.05$ was considered to indicate a statistically significant difference.

\section{Results}

CAPS mRNA level is up regulated in esophageal squamous cell cancer. The level of CAPS mRNA was upregulated in ESCC tissues. The mRNA levels of CAPS in ESCC tissues were significantly higher than those in adjacent non-cancerous tissues $(\mathrm{P}=0.0015$; Fig. 1A). In addition, CAPS mRNA levels were significantly elevated in samples of low histological grade compared with those of high histological grade $(\mathrm{P}=0.0013$; Fig. 1B). Similarly, a positive association was also detected between CAPS mRNA levels and tumor invasion $(\mathrm{P}=0.0206$; Fig. 1C).

Association between CAPS mRNA levels and clinicopathological parameters of ESCC patients. To evaluate the association between CAPS mRNA levels and clinicopathological parameters, all patients were classified as belonging to either high (cancer/normal ratio $\geq 2$ ) or low (cancer/normal ratio $<2$ ) CAPS expression groups according to the ratio of cancer tissue expression to adjacent non-cancerous tissue expression. The association between CAPS mRNA levels and patients' clinicopathological parameters is shown in Table II. The results of this analysis revealed that the level of CAPS mRNA in ESCC tissues was significantly associated with tumor invasion depth $(\mathrm{P}=0.018)$ and histological grade $(\mathrm{P}=0.017)$. However, no association was found between the CAPS mRNA level and other clinicopathological parameters, including gender, age, lymph node metastasis and TNM stage.

High CAPS protein expression in ESCC tissue is associated with poor overall survival. CAPS protein expression was further analyzed in 64 ESCC tissues and 4 corresponding adjacent non-cancerous tissues. The results of this analysis revealed that immunohistochemical staining of CAPS was predominantly observed in the cytoplasm of cancer tissues, whereas no or weak staining was found in adjacent non-cancerous tissues (Fig. 2A). The median score of tissue CAPS staining (2.5) was used as the cutoff value to divide all patients into the low CAPS expression group (n=29; Fig. 2B) and the high CAPS expression group ( $n=35$; Fig. $2 \mathrm{C}$ ). The prognostic value of CAPS expression was assessed in patients with ESCC using the Kaplan-Meier method and log-rank test. Results demonstrated that high CAPS expression was significantly associated with poorer overall survival ( $\mathrm{P}=0.0112$; Fig. 3 ).

Tissue CAPS is an independent prognostic biomarker for ESCC. Univariate and multivariate analysis was conducted 
Table II. Association of CAPS mRNA levels with clinicopathological parameters.

\begin{tabular}{|c|c|c|c|c|}
\hline \multirow[b]{2}{*}{ Clinical parameters } & \multirow[b]{2}{*}{ Total $(n=104)$} & \multicolumn{2}{|c|}{ CAPS expression } & \multirow[b]{2}{*}{ P-value } \\
\hline & & Low $(n=51)$ & $\operatorname{High}(\mathrm{n}=53)$ & \\
\hline Age, years & & & & 0.665 \\
\hline$<65$ & 61 & 31 & 30 & \\
\hline$\geq 65$ & 43 & 20 & 23 & \\
\hline Gender & & & & 0.877 \\
\hline Male & 64 & 31 & 33 & \\
\hline Female & 40 & 20 & 20 & \\
\hline Histological grade & & & & 0.017 \\
\hline Low & 20 & 5 & 15 & \\
\hline High & 84 & 46 & 38 & \\
\hline Tumor invasion depth & & & & 0.018 \\
\hline $\mathrm{T} 1 / \mathrm{T} 2$ & 53 & 32 & 21 & \\
\hline $\mathrm{T} 3 / \mathrm{T} 4$ & 51 & 19 & 32 & \\
\hline Lymph node metastasis & & & & 0.108 \\
\hline No & 74 & 40 & 34 & \\
\hline Yes & 30 & 11 & 19 & \\
\hline TNM stage & & & & 0.180 \\
\hline $\mathrm{I} / \mathrm{II}$ & 71 & 38 & 33 & \\
\hline III & 33 & 13 & 20 & \\
\hline
\end{tabular}

CAPS, calcyphosine; TNM, tumor-node-metastasis.
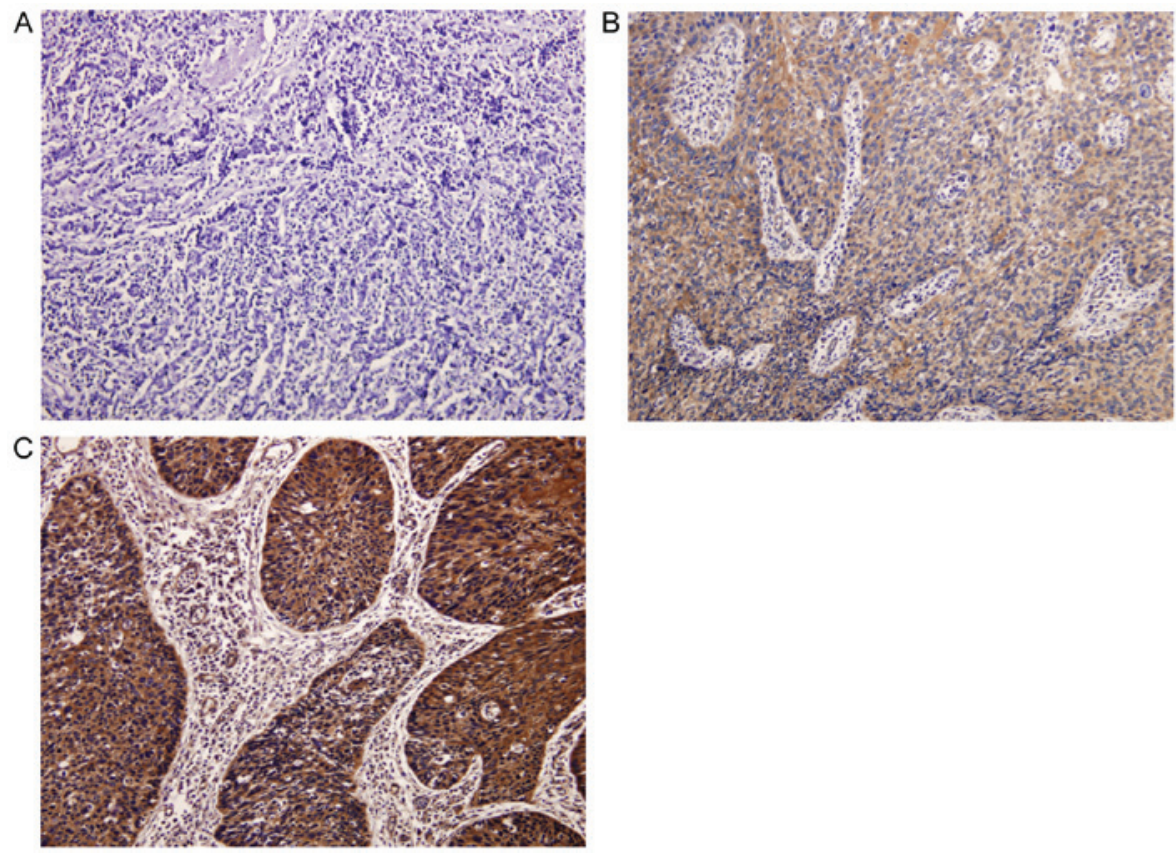

Figure 2. CAPS protein expression in ESCC tissues and adjacent normal tissues, as detected by immunohistochemistry staining. (A) Representative image of negative CAPS protein expression in healthy tissue. (B) Representative image of low CAPS expression in ESCC tissue. (C) Representative image of high CAPS expression in ESCC tissues. Original magnification, x200. CAPS, calcyphosine; ESCC, esophageal squamous cell carcinoma.

using the Cox proportional hazards model to investigate whether CAPS could serve as an independent survival predictor. In univariate analysis, histological grade [hazard ratio (HR), 2.493; 95\% confidence interval (CI), 1.117-5.564;
$\mathrm{P}=0.026]$, tumor invasion (HR, 2.483; 95\% CI, 1.193-5.167; $\mathrm{P}=0.015)$, TNM stage (HR, 3.921; 95\% CI, 1.737-8.851; $\mathrm{P}=0.001)$ and high CAPS expression (HR, 3.043; 95\% CI, 1.441-6.423; $\mathrm{P}=0.004)$ were associated with poor survival 
Table III. Cox proportional hazards regression model analysis of prognostic factors.

\begin{tabular}{|c|c|c|c|c|}
\hline \multirow[b]{2}{*}{ Variables } & \multicolumn{2}{|c|}{ Univariate analysis } & \multicolumn{2}{|c|}{ Multivariate analysis } \\
\hline & $\mathrm{HR}(95 \% \mathrm{CI})$ & P-value & $\mathrm{HR}(95 \% \mathrm{CI})$ & P-value \\
\hline Age, $\geq 65$ vs. $<65$ years & $0.961(0.466-1.980)$ & 0.914 & & \\
\hline Gender, male vs. female & $0.410(0.156-1.073)$ & 0.069 & & \\
\hline Histological grade, 3 vs. $1+2$ & $2.493(1.117-5.564)$ & 0.026 & $1.691(0.718-3.979)$ & 0.229 \\
\hline Tumor invasion depth, $\mathrm{T} 3+\mathrm{T} 4$ vs. T1+T2 & $2.483(1.193-5.167)$ & 0.015 & $1.448(0.643-3.258)$ & 0.371 \\
\hline LNM, positive vs. negative & $1.331(0.507-3.499)$ & 0.562 & & \\
\hline TNM, III vs. I+II & $3.921(1.737-8.851)$ & 0.001 & $2.748(1.191-6.341)$ & 0.018 \\
\hline CAPS expression, high vs. low & $3.043(1.441-6.423)$ & 0.004 & $2.269(1.030-4.998)$ & 0.042 \\
\hline
\end{tabular}

HR, hazard ratio; CI, confidence interval; LNM, lymph node metastasis; TNM, tumor-node-metastasis; CAPS, calcyphosine.

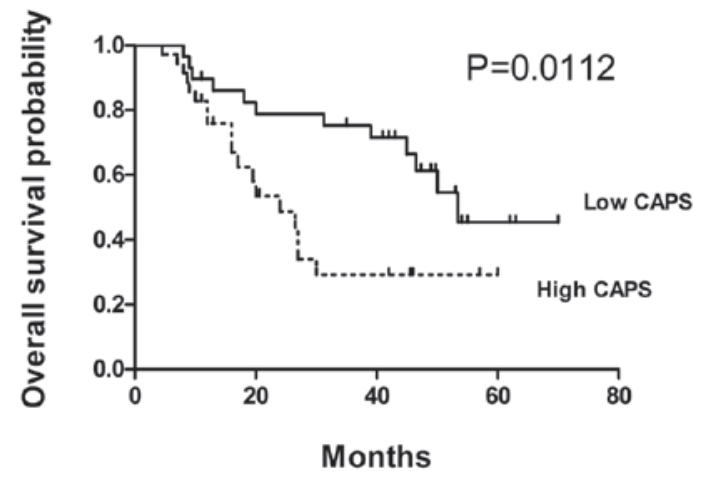

Figure 3. Kaplan-Meier curves for the overall survival analyses according to CAPS protein expression in patients with ESCC. High CAPS expression in ESCC tissues was significantly associated with poor overall survival. CAPS, calcyphosine; ESCC, esophageal squamous cell carcinoma.

of ESCC patients (Table III). Multivariate analysis revealed that TNM stage (adjusted HR, 2.748; 95\% CI, 1.191-6.341; $\mathrm{P}=0.018$ ) and high CAPS expression (adjusted HR, 2.269; 95\% CI, 1.030-4.998; $\mathrm{P}=0.042$ ) remained independent prognostic biomarkers (Table III).

\section{Discussion}

In the present study, RT-qPCR revealed that CAPS mRNA expression appeared to be frequently upregulated in ESCC tissues (Fig. 1A); the corresponding CAPS protein overexpression was also confirmed by immunohistochemical staining (Fig. 2C). To the best of our knowledge, this is the first study to demonstrate the expression profile of CAPS in ESCC. CAPS was initially identified in the canine thyroid cDNA library (3), followed by detection in certain mammals, including humans (5), cows and rabbits (6); however, it is absent from mice and five other rodents (7). A previous study revealed that the CAPS gene, consisting of 189 amino acids, is located at the p13.3 region of chromosome 19 in humans (5). Recently, attention has been directed to the association between CAPS and certain types of carcinoma. Similar to the observations of the present study, CAPS overexpressionhas been found in a range of cancer types, including ovarian cancer (20), ependymoma (14), endometrial cancer (15), lung cancer (16) and colorectal cancer (17). In a study concerning lung cancer and chronic obstructive pulmonary disease (COPD), upregulated CAPS expression was detected in lung cancer and lung cancer with COPD groups when compared with the control group, indicating that CAPS may serve as a biomarker fora lung cancer diagnosis (16).

Esophageal cancer is one of the most aggressive cancer types worldwide owing to a lack of early typical symptoms and effective non-invasive diagnostic methods (21). Despite the efforts to improve diagnostic methods and therapeutic approaches, the quality of life and overall survival time for patients with ESCC is far from satisfactory. Therefore, the identification of novel biomarkers for assisting the diagnosis and predicting the prognosis of patients with ESCC is urgently required. In recent years, substantial attention has been been paid to the identification of biomarker targets, such as p53 (22) and heat shock protein 70 (23). A previous study showed that CAPS overexpression was significantly associated with histological grade in endometrial cancer (24). Another study demonstrated that CAPS could be a novel diagnostic biomarker for patients with colorectal cancer. CAPS overexpression was positively associated with various clinicopathological parameters, including histological grade, tumor invasion, lymph node metastasis, TNM stage and distant metastasis (17). As a result, we hypothesized that the association between CAPS expression and clinicopathological parameters is cancer type-dependent. The present study examined CAPS mRNA levels in human ESCC tissues using RT-qPCR, and to the best of our knowledge, for the first time, demonstrated that CAPS mRNA expression was significantly associated with tumor invasion and histological grade in ESCC (Fig. 1B and C; Table II). These results indicated that CAPS may have a role in promoting ESCC progression.

Studies concerning ovarian cancer revealed that CAPS was overexpressed in tumor tissues compared with healthy tissue (20), and could be a predictive marker for patients with favorable tumor biology and sensitivity to treatment (13). Survival analysis has indicated that CAPS is a potential survival predictor in breast cancer patients receiving adjuvant tamoxifen (25). Previous studies also revealed that CAPS was an independent prognostic factor for endometrial 
cancer patients $(15,24)$ and colorectal cancer patients $(17)$. Therefore, we hypothesized that CAPS might be a prognostic biomarker for patients with different types of cancer. To verify this hypothesis in patients with ESCC, CAPS protein expression was detected in ESCC tissues viaimmunohistochemical staining. Results showed that 35 out of 64 tumors $(54.69 \%)$ exhibited high CAPS expression, whereas 29 (45.31\%) exhibited low CAPS expression. Kaplan-Meier analysis and the log-rank test found that ESCC patients with high CAPS expression exhibited poorer overall survival rates compared with those patients with low CAPS expression (Fig. 3). Univariate and multivariate analysis revealed that CAPS expression was an independent survival predictor for ESCC (Table III).

CAPS is involved in several types of malignant tumors. However, the molecular mechanism of CAPS function remains elusive. As a $\mathrm{Ca}^{2+}$-binding protein, CAPS may mediate its oncogenic effects through $\mathrm{Ca}^{2+}$ signaling, participating in several cellular processes, such as cell proliferation and apoptosis. Previous studies reported that alterations to intracellular $\mathrm{Ca}^{2+}$ homeostasis had a crucial role in cancer development. In a study concerning prostate cancer, data revealed that transient receptor potential cation channel subfamily $\mathrm{V}$ member 6-dependent $\mathrm{Ca}^{2+}$ influx contributed to prostate cancer development by enhancing proliferation of tumor cells and protecting them from apoptosis (26). Other studies demonstrated that higher plasma membrane channel expression and $\mathrm{Ca}^{2+}$ influx were associated with increased proliferation and tumor cell migration $(27,28)$. Therefore, we hypothesized that CAPS may promote tumorigenesis and tumor progression by disturbing intracellular $\mathrm{Ca}^{2+}$ homeostasis. Aprevious study reported that CAPS was phosphorylated and upregulated in response to thyrotropin and the cAMP cascade, and down regulated by TPA and epidermal growth factor (9). CAPS may also contribute to tumorigenesis and tumor progression through crosstalk between cAMP and EGF signals. cAMP has a vital role in the proliferation of numerous cell types $(29,30)$. Compared with normal cells, lower cAMP concentrations were found in certain tumor cells, which revealed that cAM Phas a negative role in cell proliferation (30). Additionally, intracellular cAMP levels were reported to be associated with the metastatic ability of tumor cells $(31,32)$. Epidermal growth factor signaling has been well studied with regards to controlling cell proliferation, differentiation and migration (33). Although the aforementioned signaling pathways may contribute to CAPS function in ESCC, further studies are required to clarify the exact molecular mechanism.

To the best of our knowledge, the present study demonstrated for the first time that CAPS mRNA and protein expression levels were upregulated in human ESCC. High CAPS expression was associated with histological grade and tumor invasion depth. The overall survival time of patients with high CAPS expression was significantly shorter than that of patients with low CAPS expression. These results indicate that CAPS could be a novel diagnostic indicator and an independent prognostic biomarker in ESCC. Combining the pathological diagnosis with assessment of CAPS expression levels may aid the diagnosis and predict the prognosis of patients with ESCC.

\section{Acknowledgements}

The present study was supported by the Science and Technology Innovation Team Support Plan in Universities of Henan Province (grant no. 13IRTSTHN011). The authors would like to thank Dr Xinfeng Chen (the First Affiliated Hospital of Zhengzhou University, Zhengzhou, China) for providing assistance with the experiments and $\mathrm{Mr}$ Jing $\mathrm{He}$ (Huashan Hospital Affiliated to Fudan University, Shanghai, China) for partaking in valuable discussions.

\section{References}

1. Kamangar F, Dores GM and Anderson WF: Patterns of cancer incidence, mortality, and prevalence across five continents: Defining priorities to reduce cancer disparities in different geographic regions of the world. J Clin Oncol 24: 2137-2150, 2006.

2. Xu Y, Yu X, Chen Q and Mao W: Neoadjuvant versus adjuvant treatment: Which one is better for resectable esophageal squamous cell carcinoma? World J Surg Oncol 10: 173, 2012.

3. Lecocq R, Lamy F and Dumont JE: Pattern of protein phosphorylation in intact stimulated cells: Thyrotropin and dog thyroid. Eur J Biochem 102: 147-152, 1979.

4. Lamy F, Roger PP, Lecocq R and Dumont JE: Differential protein synthesis in the induction of thyroid cell proliferation by thyrotropin, epidermal growth factor or serum. Eur J Biochem 155: 265-272, 1986.

5. El Housni H, Radulescu A, Lecocq R, Dumont JE and Christophe D: Cloning and sequence analysis of human calcyphosine complementary DNA. Biochim Biophys Acta 1352: 249-252, 1997.

6. Nemoto Y, Ikeda J, Katoh K, Koshimoto H, Yoshihara Y and Mori K: R2D5 antigen: A calcium-binding phosphoprotein predominantly expressed in olfactory receptor neurons. J Cell Biol 123: 963-976, 1993.

7. Clément S, Dumont JE and Schurmans S: Loss of calcyphosine gene expression in mouse and other rodents. Biochem Biophys Res Commun 232: 407-413, 1997.

8. Yuasa HJ, Nakatomi A, Suzuki T and Yazawa M: Genomic structure of the sponge, Halichondria okadai calcyphosine gene. Gene 298: 21-27, 2002.

9. Lecocq R, Lamy F and Dumont JE: Use of two-dimensional gel electrophoresis and autoradiography as a tool in cell biology: The example of the thyroid and the liver. Electrophoresis 11: 200-212, 1990.

10. Dong H, Li X, Lou Z, Xu X, Su D, Zhou X, Zhou W, Bartlam M and Rao Z: Crystal-structure and biochemical characterization of recombinant human calcyphosine delineates a novel EF-hand-containing protein family. J Mol Biol 383: 455-464, 2008.

11. Lefort A, Lecocq R, Libert F, Lamy F, Swillens S, Vassart G and Dumont JE: Cloning and sequencing of a calcium-binding protein regulated by cyclic AMP in the thyroid. EMBO J 8: 111-116, 1989.

12. Mehrani H, Ghanei M,Aslani J and Golmanesh L: Bronchoalveolar lavage fluid proteomic patterns of sulfur mustard-exposed patients. Proteomics Clin Appl 3: 1191-1200, 2009.

13. Partheen K, Levan K, Osterberg L and Horvath G: Expression analysis of stage III serous ovarian adenocarcinoma distinguishes a sub-group of survivors. Eur J Cancer 42: 2846-2854, 2006.

14. de Bont JM, den Boer ML, Kros JM, Passier MM, Reddingius RE, Smitt PA, Luider TM and Pieters R: Identification of novel biomarkers in pediatric primitive neuroectodermal tumors and ependymomas by proteome-wide analysis. J Neuropathol Exp Neurol 66: 505-516, 2007.

15. Li Z, Min W, Huang C, Bai S, Tang $M$ and Zhao X: Proteomics-based approach identified differentially expressed proteins with potential roles in endometrial carcinoma. Int $\mathrm{J}$ Gynecol Cancer 20: 9-15, 2010.

16. Pastor MD, Nogal A, Molina-Pinelo S, Meléndez R, Salinas A, González De la Peña M, Martín-Juan J, Corral J, García-Carbonero R, Carnero A and Paz-Ares L: Identification of proteomic signatures associated with lung cancer and COPD. J Proteomics 89: 227-237, 2013. 
17. Shao W, Wang Q, Wang F, Jiang Y, Xu M and Xu J: Abnormal expression of calcyphosine is associated with poor prognosis and cell biology function in colorectal cancer. Onco Targets Ther 9: 477-487, 2016.

18. Rice TW, Blackstone EH and Rusch VW: 7th edition of the AJCC cancer staging manual: Esophagus and esophagogastric junction. Ann Surg Oncol 17: 1721-1724, 2010.

19. Livak KJ and Schmittgen TD: Analysis of relative gene expression data using real-time quantitative PCR and the 2(-Delta Delta C(T)) method. Methods 25: 402-408, 2001.

20. Skubitz AP, Pambuccian SE, Argenta PA and Skubitz KM Differential gene expression identifies subgroups of ovarian carcinoma. Transl Res 148: 223-248, 2006.

21. Tentzeris V, Lake B, Cherian T, Milligan J and Sigurdsson A: Poor awareness of symptoms of oesophageal cancer. Interact Cardiovasc Thorac Surg 12: 32-34, 2011.

22. Shimada H, Takeda A, Arima M, Okazumi S, Matsubara H, Nabeya Y, Funami Y, Hayashi H, Gunji Y, Suzuki T, et al: Serum p53 antibody is a useful tumor marker in superficial esophageal squamous cell carcinoma. Cancer 89: 1677-1683, 2000.

23. Fujita Y, Nakanishi T, Miyamoto Y, Hiramatsu M, Mabuchi H, Miyamoto A, Shimizu A, Takubo $T$ and Tanigawa N: Proteomics-based identification of autoantibody against heat shock protein 70 as a diagnostic marker in esophageal squamous cell carcinoma. Cancer Lett 263: 280-290, 2008

24. Li Z, Huang C, Bai S, Pan X, Zhou R, Wei Y and Zhao X: Prognostic evaluation of epidermal fatty acid-binding protein and calcyphosine, two proteins implicated in endometrial cancer using a proteomic approach. Int J Cancer 123: 2377-2383, 2008.

25. Johansson HJ, Sanchez BC, Forshed J, Stål O, Fohlin H, Lewensohn R, Hall P, Bergh J, Lehtiö J and Linderholm BK Proteomics profiling identify CAPS as a potential predictive marker of tamoxifen resistance in estrogen receptor positive breast cancer. Clin Proteomics 12: 8, 2015.
26. Raphaël M, Lehen'kyi V, Vandenberghe M, Beck B, Khalimonchyk S, Vanden Abeele F, Farsetti L, Germain E, Bokhobza A, Mihalache A, et al: TRPV6 calcium channel translocates to the plasma membrane via Orail-mediated mechanism and controls cancer cell survival. Proc Natl Acad Sci USA 111: E3870-E3879, 2014.

27. Déliot $\mathrm{N}$ and Constantin B: Plasma membrane calcium channels in cancer: Alterations and consequences for cell proliferation and migration. Biochim Biophys Acta 1848: 2512-2522, 2015.

28. Di Virgilio F: Purines, purinergic receptors, and cancer. Cancer Res 72: 5441-5447, 2012.

29. Dumont JE, Jauniaux JC and Roger PP: The cyclic AMP-mediated stimulation of cell proliferation. Trends Biochem Sci 14: 67-71, 1989.

30. MacManus JP, Whitfield JF, Boynton AL and Rixon RH: Role of cyclic nucleotides and calcium in the positive control of cell proliferation. Adv Cyclic Nucleotide Res 5: 719-734, 1975.

31. Sheppard JR, Koestler TP, Corwin SP, Buscarino C, Doll J, Lester B, Greig RG and Poste G: Experimental metastasis correlates with cyclic AMP accumulation in B16 melanoma clones. Nature 308: 544-547, 1984.

32. Chen TC, Hinton DR, Zidovetzki R and Hofman FM: Up-regulation of the cAMP/PKA pathway inhibits proliferation, induces differentiation, and leads to apoptosis in malignant gliomas. Lab Invest 78: 165-174, 1998.

33. Yarden Y and Pines G: The ERBB network: At last, cancer therapy meets systems biology. Nat Rev Cancer 12: 553-563, 2012. 